\title{
Tumor necrosis factor superfamily member APRIL contributes to fibrotic scar formation after spinal cord injury
}

\author{
Lucy H. Funk', Amber R. Hackett ${ }^{1}$, Mary Bartlett Bunge $e^{1,2,3}$ and Jae K. Lee ${ }^{1,4^{*}}$
}

\begin{abstract}
Background: Fibrotic scar formation contributes to the axon growth-inhibitory environment that forms following spinal cord injury (SCl). We recently demonstrated that depletion of hematogenous macrophages led to a reduction in fibrotic scar formation and increased axon growth after SCl. These changes were associated with decreased TNFSF13 (a proliferation inducing ligand (APRIL)) expression, but the role of APRIL in fibrotic scar formation after SCI has not been directly investigated. Thus, the goal of this study was to determine the role of APRIL in fibrotic scar formation after SCl.

Methods: APRIL knockout and wild-type mice received contusive SCl and were assessed for inflammatory cytokine/ chemokine expression, leukocyte infiltration, fibrotic scar formation, axon growth, and cell proliferation.

Results: Expression of APRIL and its receptor BCMA is increased following SCl, and genetic deletion of APRIL led to reduced fibrotic scar formation and increased axon growth. However, the fibrotic scar reduction in APRIL KO mice was not a result of changes in fibroblast or astrocyte proliferation. Rather, APRIL knockout mice displayed reduced TNFa and CCL2 expression and less macrophage and B cell infiltration at the injury site.
\end{abstract}

Conclusions: Our data indicate that APRIL contributes to fibrotic scar formation after SCl by mediating the inflammatory response.

Keywords: TNFSF13, Glial scar, Cell proliferation, Fibrosis, BAFF, TACl, BCMA, BAFF-R

\section{Introduction}

Developing successful therapies for spinal cord injury (SCI) is a formidable medical challenge in part because of the axonal growth-inhibitory environment that develops following injury characterized by a glial and fibrotic scar [1]. Whereas the glial scar has been an important research focus for many years, less attention has been given to the fibrotic scar. Our recent work demonstrated that hematogenous macrophages are important for the formation of the fibrotic scar [2]. Additionally, reduced fibrotic scar formation after macrophage depletion was associated with decreased expression of tumor necrosis factor superfamily member

\footnotetext{
*Correspondence: JLee22@med.miami.edu

'Department of Neurological Surgery, Miami Project to Cure Paralysis,

University of Miami School of Medicine, Miami, FL 33136, USA

${ }^{4}$ University of Miami School of Medicine, 1095 NW 14th Terrace, LPLC 4-19,

Miami, FL 33136, USA

Full list of author information is available at the end of the article
}

13 (TNFSF13), also known as a proliferation inducing ligand (APRIL). Thus, our goal for this study was to determine if APRIL is necessary in the formation of the fibrotic scar following contusive SCI.

Past research on APRIL has focused mostly on its role in cancer and B cell survival and proliferation [3]. APRIL has been shown to be elevated in several diseases such as rheumatoid arthritis [4], systemic lupus erythematosus [5], multiple sclerosis [6], and schizophrenia [7]. Macrophages [8, 9], B cells, and activated T cells [10] express APRIL, and APRIL can interact with transmembrane activator and CAML interactor (TACI), B cell maturation antigen (BCMA), and heparan sulfate proteoglycan (HSPG) receptors [11-14]. These receptors are shared with another closely related tumor necrosis factor (TNF) superfamily member B cell-activating factor (BAFF, TNFSF13b) [12, 13]. Many of APRIL's functions are attributed to activating the nuclear factor 
kappa-light-chain-enhancer of activated B cells (NF- $\mathrm{kB}$ ) pathway which in turn can induce APRIL expression $[15,16]$. Information on APRIL in the CNS is limited. APRIL has been shown to be expressed by hippocampal pyramidal cells and astrocytes; microglia have been shown to express TACI [17-19]. However, the role of APRIL following traumatic CNS injury has not been studied.

To test the hypothesis that APRIL is necessary for fibrotic scar formation after SCI, we performed SCI in APRIL knockout (KO) mice. We found that APRIL expression is upregulated following SCI and genetic deletion of APRIL attenuates the inflammatory response and reduces fibrotic scar area. To our knowledge, this is the first study to directly investigate the role of APRIL after traumatic CNS injury in vivo.

\section{Materials and methods}

\section{Surgery and behavioral assessment}

TNFSF13 KO mice in a C57BL/6 genetic background were kindly donated by Dr. Eckhard Podack. Wild-type C57BL/6 mice served as controls. Eight- to twelve-week old female mice underwent a moderate contusive SCI as previously described [20]. Briefly, anesthetized mice (ketamine/xylazine, $100 \mathrm{mg} / 15 \mathrm{mg} / \mathrm{kg}$ i.p.) received a T8 laminectomy followed by a moderate contusive SCI (65 kDynes) using an Infinite Horizon Impactor (Precision Systems and Instrumentation, LLC). Postoperative treatment for the first week included twice daily injections of Lactated Ringer's solution $(1 \mathrm{ml})$, an antibiotic (Baytril, $10 \mathrm{mg} / \mathrm{kg}$ ), and an analgesic (buprenorphine, $0.05 \mathrm{mg} / \mathrm{kg}$ ) subcutaneously. Twice daily bladder expressions continued for the duration of the study. Locomotor recovery was assessed using the Basso Mouse Scale [21] open-field test at 1 day and weekly after injury. Only mice with a BMS score of 0 or 1 at 1 day after injury were included in the study. To identify proliferating cells, $50 \mathrm{mg} / \mathrm{kg} \mathrm{5-}$ ethynyl-2'-deoxyuridine (EdU) (Invitrogen A10044) was injected intraperitoneally on days 3,4 , and 5 following SCI. All procedures were in accordance with University of Miami Institutional Animal Care and Use Committee (IACUC) and National Institutes of Health (NIH) guidelines.

\section{Gene expression analysis}

Mice were anesthetized as described above and perfused transcardially with cold Dulbecco's phosphate-buffered saline (DPBS, Gibco 14190-144). Naïve or wild-type (WT) injured mice were used as controls. A 4-mm section of the spinal cord injury site, or the corresponding T8 spinal cord in naïve controls, was dissected and then homogenized for RNA extraction using the Qiagen RNeasy Plus Micro kit (74034). Subsequently, cDNA was synthesized using the Advantage RT-for-polymerase chain reaction (PCR) kit (Clontech 639506). cDNA (0.5 $\mu \mathrm{g}$ per sample) was added to $\mathrm{RT}^{2}$ SYBR Green Mastermix and PCR performed using Eppendorf Mastercycler ep realplex. Primer sequences were as follows $\left(5^{\prime}-3^{\prime}\right)$ : APRIL (forward AATTCTCCTGAGGCTAGGGGG; reverse AG GACATCAGGACTCTGCTCC), BAFF (forward CGAC ACGCCGACTATACGAA; reverse GGTCCGTGTATA GAACCTGGC), BCMA (forward ACTTGCGATGTTC CAACCCT; reverse ATCCGTCAAGCTGACCTGG), TA CI (forward ACGTGTAGCTTCTGCTTCCC; reverse CG CCTACTGTTGCACGCATA), BAFF-R (forward CCAG TGCAATCAGACCGAGT; reverse GGGTTTCTGAG GAGGGTACAA), GAPDH (forward TGGCCTTCCG TGTTCCTAC; reverse GAGTTGCTGTTGAAGTCG), TNF (forward AGGCACTCCCCCAAAAGATG; reverse TCACCCCGAAGTTCAGTAGAC), CCL2 (forward CCC CACTCACCTGCTGCTAC; reverse CCTGCTGCTGGT GATTCTCTT), IL-1 $\beta$ (forward CTTCAAATCTCACAG CAGCACATC; reverse CCACGGGAAAGACACAGGT AG), CXCL10 (forward GCCGTCATTTTCTGCCTCA TCCT; reverse CTCATTCTCACTGGCCCGTCATC), CCL5 (forward TGCCCACGTCAAGGAGTATTTCTA; reverse TGGCGGTTCCTTCGAGTGACAA), and IL-6 (forward AACCACGGCCTTCCCTACTTCA; reverse TC ATTTCCACGATTTCCCAGAG ).
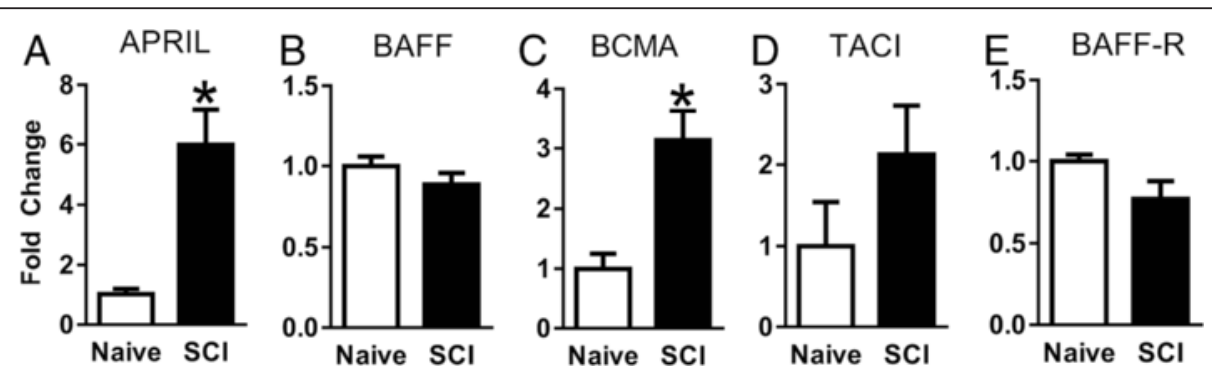

Fig. 1 mRNA expression of APRIL, BAFF, and their receptors at 2 weeks after contusive spinal cord injury. In the injured spinal cord, APRIL expression is significantly increased whereas its related ligand BAFF is unaltered (a-b). Of the APRIL family receptors, BCMA (c) expression is significantly increased but TACl (d) and BAFF-R (e) are not changed. $n=5$ per group (biological replicates). Normalized to average of naïve for each gene. ${ }^{*} p<0.01$ compared to naïve. Student's $t$ test 


\section{Histology}

Mice were anesthetized and perfused transcardially with $4 \%$ paraformaldehyde 2 weeks post-SCI. Spinal cords were dissected and post-fixed for $2 \mathrm{~h}$ before placing in $30 \%$ sucrose overnight. An $8-\mathrm{mm}$ spinal segment centered at the injury site was embedded in OCT compound (Tissue-Tek) and sectioned sagittally on a cryostat into $16-\mu \mathrm{m}$ serial sections. Sections were subsequently immunostained with primary antibodies for glial fibrillary acidic protein (GFAP) (Invitrogen 130300, 1:500), Neurofilament medium (EnCor Biotechnology, RPCA-NF-M, 1:500) and platelet-derived growth factor receptor beta (PDGFR $\beta$ ) (Abcam plc, ab32570, 1:200) in PBS-0.3 \% Triton X-100. The sections were then
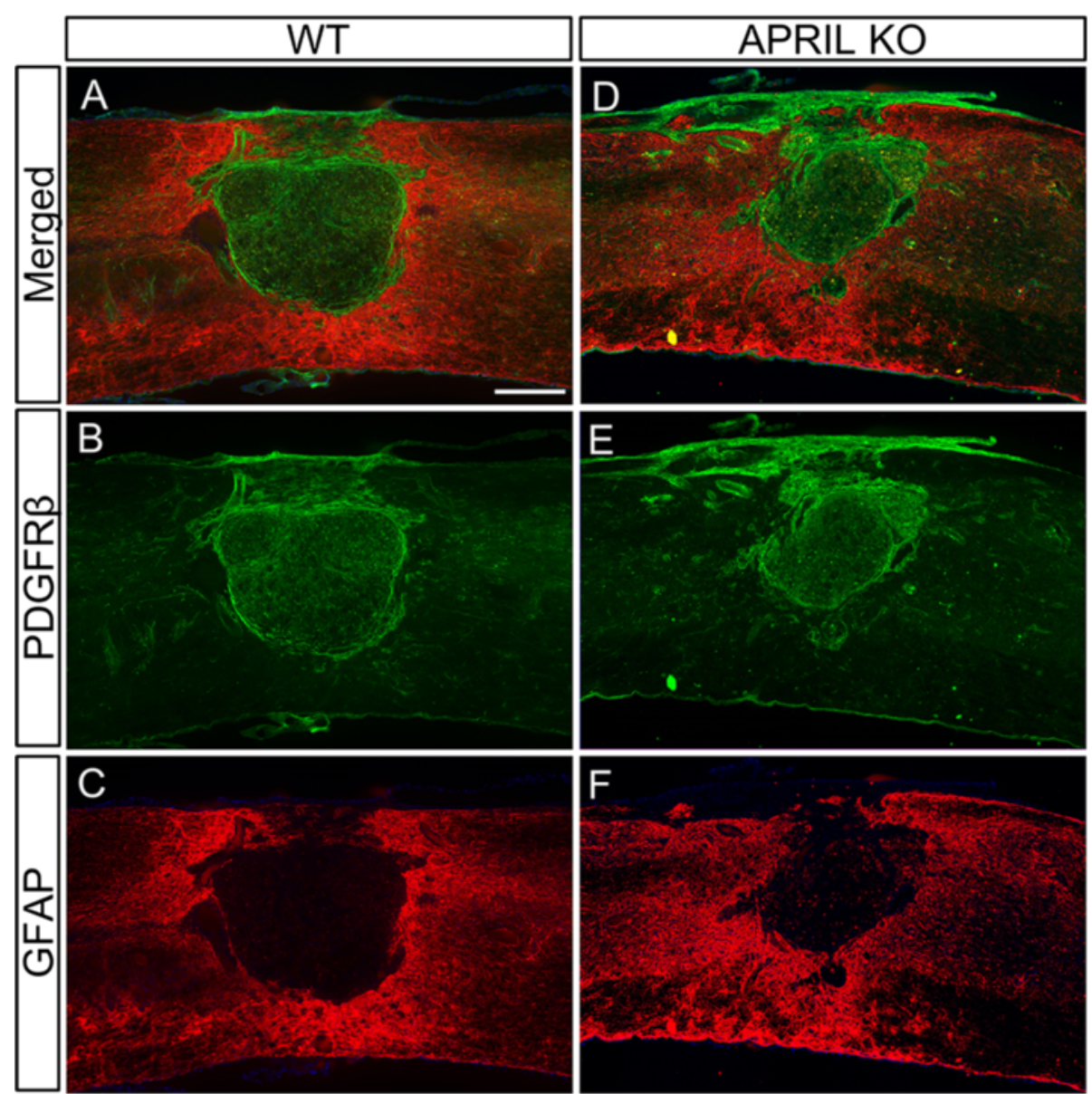

G
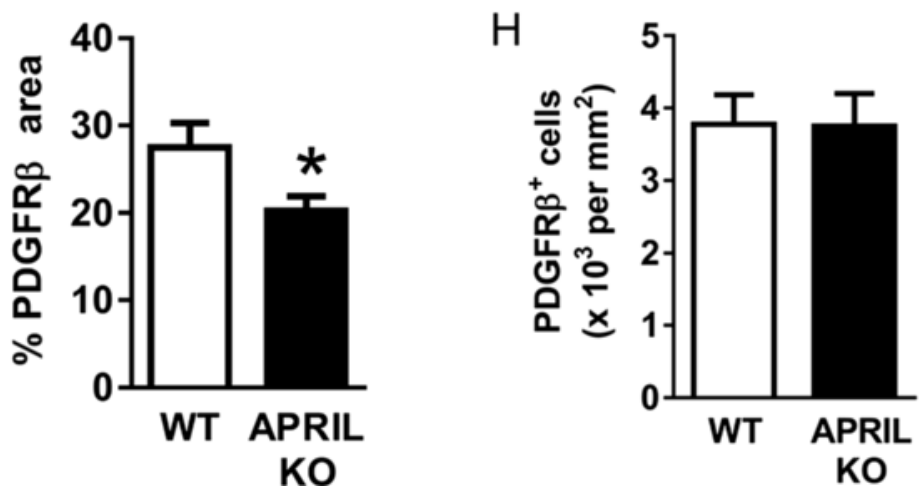

Fig. 2 APRIL KO mice have a reduced fibrotic scar area after SCl. Two weeks following SCl, the fibrotic scar size was assessed in APRIL KO (d-f, $n=9$ ) and WT ( $\mathbf{a}-\mathbf{c}, n=11)$ mice by evaluating the percent area of PDGFR $\beta$ signal within a 2-mm region centered around the injury site (g). The density of PDGFR ${ }^{+}$cells was not different between WT and KO mice (h). Representative images from a WT and APRIL KO animal are shown with GFAP in red and PDGFR $\beta$ in green. $n=$ biological replicates; ${ }^{*} p<0.05$ compared to WT. Student's $t$ test. Scale bar $=250 \mu \mathrm{m}$ 
incubated with the corresponding Alexa Fluor secondary antibodies (Invitrogen, 1:500). Sections were mounted in VECTASHIELD containing DAPI (Vector Laboratories), and images were collected with a Nikon Eclipse Ti fluorescent microscope. EdU labeling was visualized using Life Technologies Click-iT EdU imaging kit (Life Technologies, C10340).

\section{Quantification}

Quantification of immunohistochemical images was performed using ImageJ by observers blinded to the experimental groups. The fibrotic scar size was measured by tracing the PDGFR $\beta^{+}$area within a $2-\mathrm{mm}$ segment of the spinal cord centered on the injury site. The PDGFR $\beta^{+}$area was expressed as a percentage of the area of the 2-mm spinal cord segment evaluated. To quantify the number of neurofilament ${ }^{+}$axons and $\mathrm{EdU}^{+}$fibroblasts, $50-\mu \mathrm{m}$ square grids were generated over the injury site and every sixth square in the $\mathrm{GFAP}^{-}$region was quantified. To quantify the EdU ${ }^{+}$astrocytes, every third square in the grid within the $250-\mu \mathrm{m}$ border region of the glial scar was evaluated. For each animal, sections including the injury epicenter and two adjacent sagittal sections spaced $128 \mu \mathrm{m}$ apart were quantified, and the counts from each section were averaged.

\section{Flow cytometry}

A cell suspension of the spinal cord 1 week following injury underwent myelin removal (myelin removal beads, Miltenyi Biotec). Subsequently, cells were resuspended in 100- $\mu$ l FACS buffer and Fc blocked with anti-mouse
CD16/32 (BioLegend, 1:200) for $10 \mathrm{~min}$ on ice and were then incubated for $30 \mathrm{~min}$ at $4{ }^{\circ} \mathrm{C}$ with anti-CD45-APC/ Cy7, anti-CD11b-APC, anti-B220-FITC, and anti-CD3$\mathrm{PE} / \mathrm{Cy} 7$. Cell suspensions were analyzed as previously described [2]. Cell numbers were quantified using 123count ebeads (eBioscience).

\section{Results}

The expression of APRIL, BAFF, and their receptors in the spinal cord was evaluated using qRT-PCR following SCI. Expression of APRIL was increased at 14 days after SCI (Fig. 1a). The APRIL receptor BCMA, but not $\mathrm{TACI}$, was also significantly increased following SCI (Fig. 1c, d). However, expression of the related ligand BAFF and BAFF-R was not altered following SCI (Fig. 1b, e). To evaluate the role of APRIL in the formation of the fibrotic scar, we measured PDGFR $\beta^{+}$areas at 2 weeks after SCI. We previously demonstrated that PDGFR $\beta$ is a reliable marker of fibroblasts at the SCI site [20]. We chose the 2-week time point because this is when the scar starts to mature and take its final shape. In both WT and APRIL KO mice, PDGFR $\beta^{+}$fibroblasts were present throughout the $\mathrm{GFAP}^{-}$region of the injury site with a dense boundary apposing the astroglial scar (Fig. 2a-f). However, at 2 weeks after SCI, the PDGFR $\beta^{+}$ area in APRIL KO mice was significantly reduced (Fig. $2 \mathrm{~d}-\mathrm{g}$ ), whereas the fibroblast density remained the same (Fig. 2h), suggesting that APRIL contributes to fibrotic scar formation after SCI. To determine the effect of this reduced fibrotic scar on axon growth, we measured the number of neurofilament-positive axons in the

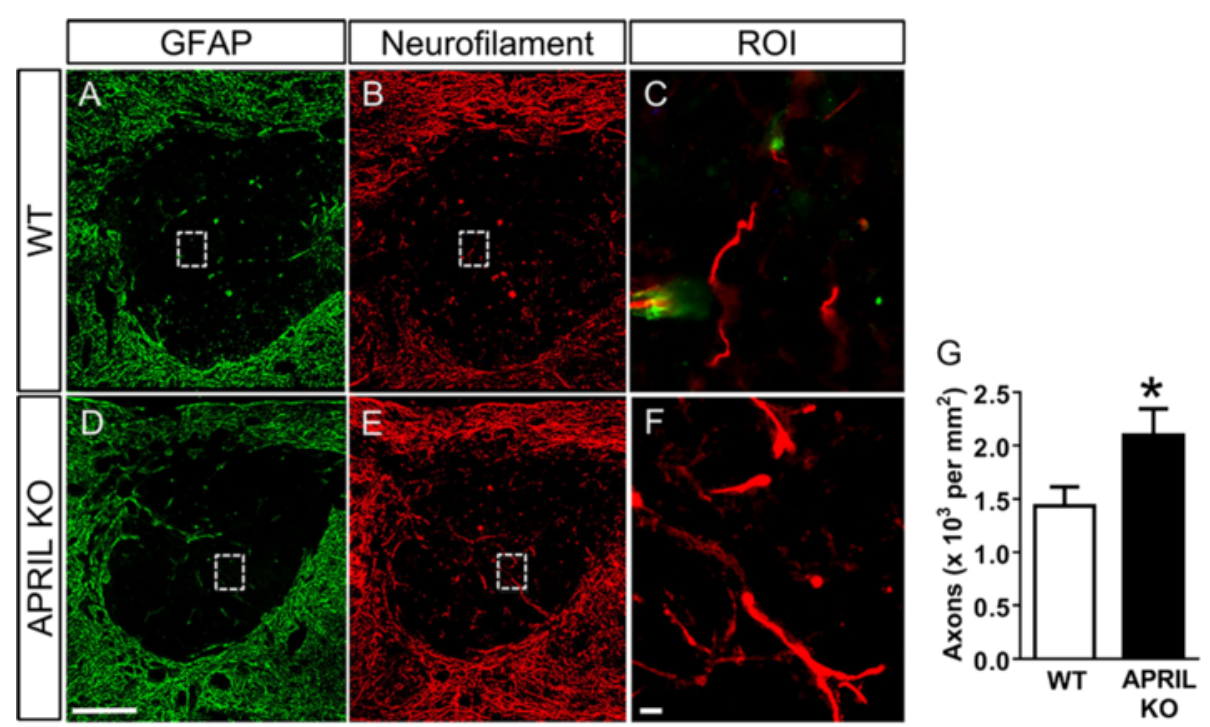

Fig. 3 APRIL KO mice have an increased number of axons in the injury site 2 weeks following SCI. Neurofilament ${ }^{+}$axons (red) in the GFAP (green)-negative region were compared between WT (a-c) and APRIL KO (d-f) mice 2 weeks following injury. $\mathbf{c}$, $\mathbf{f}$ Magnified images of dotted area (region of interest $(\mathrm{ROI})$ ). Quantifications are shown in $\mathbf{g}\left(n=8\right.$ biological replicates). Scale bar $=250 \mu \mathrm{m}(\mathbf{a}, \mathbf{b}, \mathbf{d}, \mathbf{e}), 10 \mu \mathrm{m}(\mathbf{c}, \mathbf{f}) .{ }^{*} p<0.05$ compared to WT. Student's $t$ test 
fibrotic scar region. APRIL KO mice had more axons in the fibrotic scar compared to wild-type mice, suggesting that genetic deletion of APRIL promotes axon growth by reducing the fibrotic scar (Fig. 3).

To determine if the reduced fibrotic scar size was due to decreased fibroblast proliferation, EdU was injected on days 3,4 , and 5 following injury. These time points have been previously shown to be the peak of glial cell proliferation as well as when fibroblasts start to appear after SCI [20, 22]. Two weeks after SCI, there was no difference between WT (Fig. 4a-i) and APRIL KO (Fig. 4j) animals in the percentage of EdU ${ }^{+}$fibroblasts (Fig. 4k), demonstrating that the smaller fibrotic scar was not due to a reduction in fibroblast proliferation in APRIL KO mice. To determine if the smaller fibrotic scar could be due to an increased glial scar size resulting from increased astrocyte proliferation, the number of $\mathrm{EdU}^{+} \mathrm{GFAP}^{+}$cells was counted in the $250-\mu \mathrm{m}$ border of the glial scar (Fig. 4). The number of EdU ${ }^{+}$astrocytes in the glial scar border was not different between WT and APRIL KO mice (Fig. 4l), suggesting that astrocyte proliferation was not significantly affected after genetic deletion of APRIL after SCI. Taken together, our data indicate that the reduced fibrotic scar size is not due to changes in fibroblast or astrocyte proliferation after SCI.

We had previously demonstrated that macrophages are an important mediator of fibrotic scar formation after SCI [2]. Thus, to consider the possibility that the reduced fibrotic scar in APRIL KO mice was an indirect result of the effect of APRIL on macrophage recruitment, we assessed leukocyte infiltration in APRIL KO mice. At 7 days after SCI, we dissociated the injury site tissue from APRIL KO and wild-type mice and performed flow cytometry to assess the number of macrophages $\left(\mathrm{CD} 45^{\text {hi }} \mathrm{CD} 11 \mathrm{~b}^{+}\right)$, microglia $\left(\mathrm{CD} 45^{\text {low }} \mathrm{CD} 11 \mathrm{~b}^{+}\right), \mathrm{B}$ cells $\left(\mathrm{B}_{2} 20^{+}\right)$, and $\mathrm{T}$ cells $\left(\mathrm{CD}^{+}\right)$. We chose the 7 -day time point because this is the peak of macrophage infiltration [23]. Whereas the percentages of each cell type

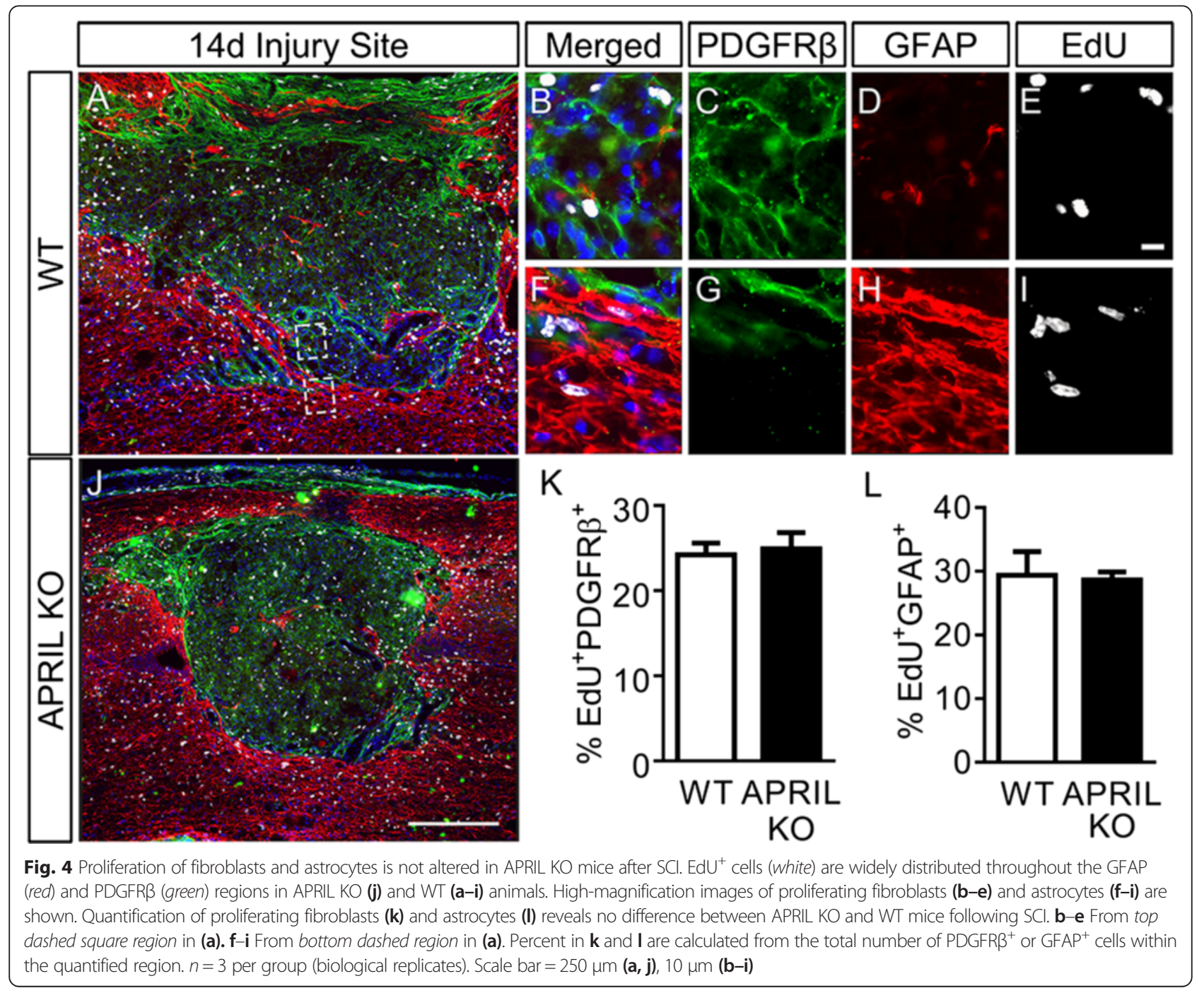



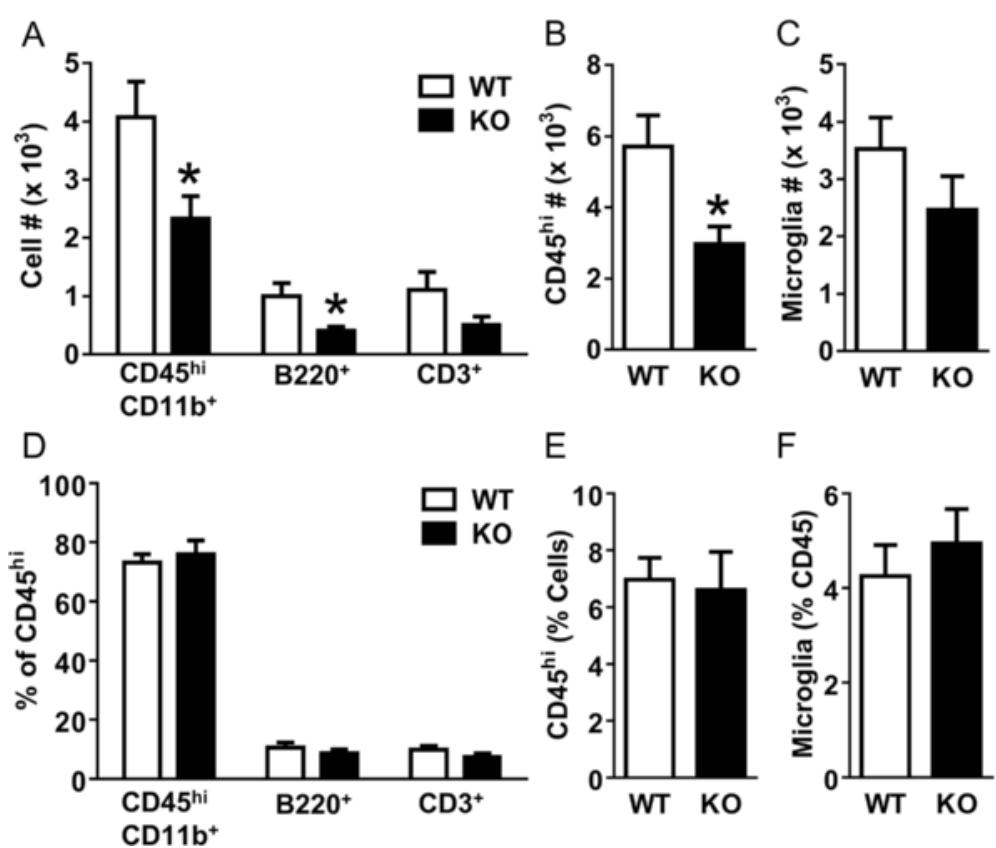

$\mathrm{E}$

$\mathrm{F}$
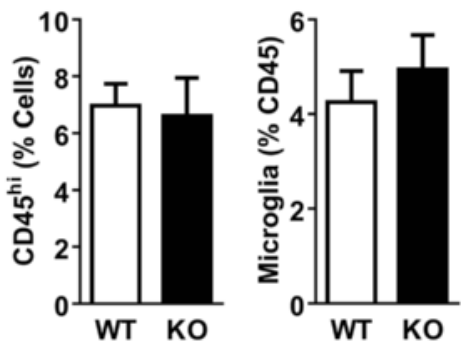

Fig. 5 The number of infiltrating leukocytes is decreased in APRIL KO mice 1 week following SCI. The number of infiltrating macrophages $\left(\mathrm{CD} 45^{\text {hi }} \mathrm{CD} 1 \mathrm{~b}^{+}\right)$and B cells $\left(\mathrm{B} 220^{+}\right)$were significantly reduced following SCI in APRIL KO mice $(n=7)$ compared to WT controls $(n=10)(\mathbf{a})$. This translated to a reduction in the overall number of infiltrating leukocytes (b), whereas the number of microglia remained similar between WT and $\mathrm{KO}$ mice (c). However, the percentages of each cell type, including microglia, were not different (d-f). $n=$ biological replicates. ${ }^{*} p<0.05$ compared to WT. Student's $t$ test

were similar between wild-type and APRIL KO mice (Fig. 5d-f), the number of macrophages and B cells was significantly reduced (Fig $5 \mathrm{a}-\mathrm{c}$ ). This translated into a reduction in the overall leukocyte number $\left(\mathrm{CD} 45^{\mathrm{hi}}\right)$ at the injury site (Fig. 5b). However, the number of microglia and $\mathrm{T}$ cells was not different between the two groups. Therefore, the reduced fibrotic scar in APRIL $\mathrm{KO}$ mice was associated with reduced macrophage and $\mathrm{B}$ cell infiltration after $\mathrm{SCI}$.

To determine whether the reduced leukocyte infiltration could be due to altered cytokine/chemokine expression acutely after injury in APRIL KO mice, we used qRT-PCR to assess the expression of multiple inflammatory cytokines/chemokines at 1 day after SCI, which is considered the peak of inflammatory cytokine/chemokine expression [24]. We found that APRIL KO mice have reduced expression of TNF $\alpha$ and CCL2 compared to wild-type controls (Fig. 6a, b), indicating that APRIL contributes to the acute expression of inflammatory cytokines/chemokines after SCI and raising the possibility that this reduced acute inflammatory response may have eventually led to

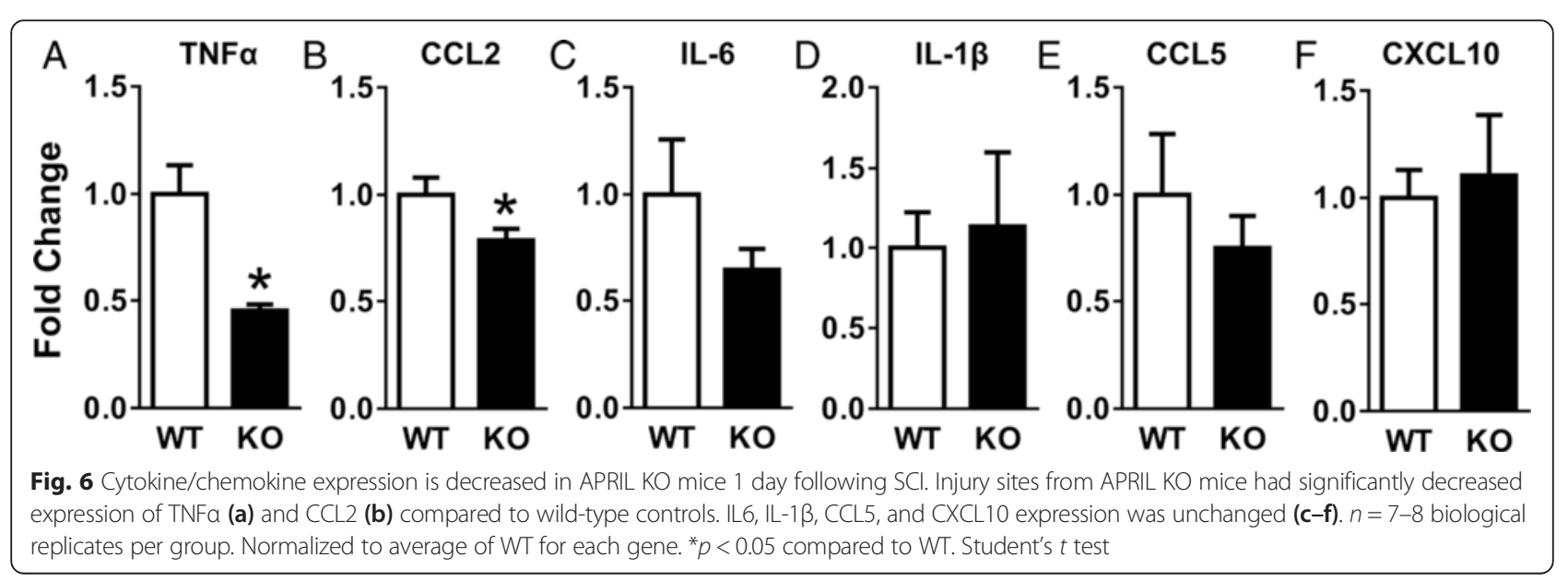


reduced leukocyte infiltration that resulted in attenuated fibrotic scar formation.

\section{Discussion}

Our goal in this study was to test the role of APRIL in fibrotic scar formation following SCI. We demonstrated that APRIL and BCMA expression is increased following SCI and that genetic deletion of APRIL leads to a reduced fibrotic scar area that is associated with increased axonal growth. This reduction, however, was not due to altered proliferation of fibroblasts or astrocytes at the injury site, which was surprising given the known role of APRIL in cancer metastasis and reports of APRIL affecting proliferation of fibroblast-like synoviocytes and astrocytes $[18,25]$. In addition, the density of fibroblasts was not different between WT and APRIL KO mice, suggesting that reduced fibrotic areas was not due to compaction of this region, but rather to a reduced overall number of fibroblasts recruited to the injury site.

Thus, we pursued the possibility that rather than acting directly on fibroblasts, APRIL could be mediating fibrotic scar formation indirectly through macrophages. Indeed, we found significantly reduced macrophage number at the injury site of APRIL KO mice. In addition, we also found a reduced number of B cells, which was expected given the well-known role of APRIL in regulating $\mathrm{B}$ cell proliferation. This reduction in leukocyte infiltration was associated with reduced TNF $\alpha$ and CCL2 expression in APRIL KO mice, suggesting that the reduced leukocyte infiltration was due, at least in part, to the regulation of the acute inflammatory response by APRIL. Taken together, our data suggest that APRIL regulates the acute expression of pro-inflammatory cytokines that leads to recruitment of leukocytes to form the fibrotic scar after SCI. However, we cannot rule out the possibility that these processes occur in parallel with direct effects of APRIL on leukocyte infiltration and/or fibrotic scar formation.

Our finding that TNF $\alpha$ and CCL2 expression is decreased 1 day after SCI in APRIL KO mice suggests that APRIL is a regulator of the acute inflammatory response. Since macrophages and lymphocytes are not a major cellular component of the injury site at this early time point, the most likely sources of APRIL are neutrophils, microglia, and/or astrocytes. The mechanism by which APRIL is expressed by these cells regulates cytokine expression after SCI remains to be determined. In conclusion, our data indicate that APRIL contributes to fibrotic scar formation after SCI by mediating the acute inflammatory response and recruitment of leukocytes.
Ethics approval and consent to participate

All animal procedures were in accordance with University of Miami IACUC and NIH guidelines. There are no human participants, data, or tissue in this study.

\section{Availability of data and materials}

All datasets on which the conclusions of the manuscript rely are presented in this manuscript.

\section{Abbreviations \\ APRIL: a proliferation inducing ligand; BAFF: B cell-activating factor; BAFF-R: B cell-activating factor receptor; BCMA: B cell maturation antigen; BMS: Basso Mouse Scale; EdU: 5-ethynyl-2'-deoxyuridine; HSPG: heparan sulfate proteoglycan; KO: knockout; NF-kB: nuclear factor kappa-light-chain-enhancer of activated B cells; PCR: polymerase chain reaction; PDGFRß: platelet-derived growth factor receptor beta; ROI: region of interest; SCl: spinal cord injury; TACl: transmembrane activator and CAML interactor; TNF: tumor necrosis factor; TNFSF: tumor necrosis factor superfamily; WT: wild-type.}

\section{Competing interests}

The authors declare that they have no competing interests.

\section{Authors' contributions}

LF designed and performed the experiments and wrote the manuscript. JL designed the experiments and wrote the manuscript. $\mathrm{AH}$ performed the experiments, and MBB contributed to writing the manuscript. All authors read and approved the final manuscript.

\section{Acknowledgements}

We thank Yadira Salgueiro and Shaffiat Karmally for their technical assistance. We thank Dr. Roberta Brambilla, Han Gao, and Dr. Oliver Umland for assistance with flow cytometry experiments. We thank Dr. Eckhard Podack, Department of Microbiology and Immunology, for the APRIL KO mice.

\section{Funding}

This study was funded by NINDS R01NS081040 (JKL), R21NS082835 (JKL), R01NS009923 (MBB), The Miami Project to Cure Paralysis, and the Buoniconti Fund. Other than financial support and/or review of the grant applications, the funding bodies did not play any role in the design of the study, collection, analysis, and interpretation of data, and in writing the manuscript.

\section{Author details}

${ }^{1}$ Department of Neurological Surgery, Miami Project to Cure Paralysis, University of Miami School of Medicine, Miami, FL 33136, USA. ²Department of Cell Biology, University of Miami School of Medicine, Miami, FL 33136, USA. ${ }^{3}$ Department of Neurology, University of Miami School of Medicine, Miami, FL 33136, USA. ${ }^{4}$ University of Miami School of Medicine, 1095 NW 14th Terrace, LPLC 4-19, Miami, FL 33136, USA.

Received: 1 April 2016 Accepted: 14 April 2016

Published online: 20 April 2016

\section{References}

1. Cregg JM, DePaul MA, Filous AR, Lang BT, Tran A, Silver J. Functional regeneration beyond the glial scar. Exp Neurol. 2014;253:197-207.

2. Zhu Y, Soderblom C, Krishnan V, Ashbaugh J, Bethea JR, Lee JK. Hematogenous macrophage depletion reduces the fibrotic scar and increases axonal growth after spinal cord injury. Neurobiol Dis. 2015;74:114-25.

3. Planelles L, Medema JP, Hahne M, Hardenberg G. The expanding role of APRIL in cancer and immunity. Curr Mol Med. 2008:8:829-44.

4. Weldon AJ, Moldovan I, Cabling MG, Hernandez EA, Hsu S, Gonzalez J, Parra A, Benitez A, Daoud N, Colburn K, Payne KJ. Surface APRIL is elevated on myeloid cells and is associated with disease activity in patients with rheumatoid arthritis. J Rheumatol. 2015;42:749-59.

5. Boghdadi G, Elewa EA. Increased serum APRIL differentially correlates with distinct cytokine profiles and disease activity in systemic lupus erythematosus patients. Rheumatol Int. 2014;34:1217-23. 
6. Wang H, Wang K, Zhong X, Qiu W, Dai Y, Wu A, Hu X. Cerebrospinal fluid BAFF and APRIL levels in neuromyelitis optica and multiple sclerosis patients during relapse. J Clin Immunol. 2012;32:1007-11.

7. Catts VS, Weickert CS. Gene expression analysis implicates a death receptor pathway in schizophrenia pathology. PLoS One. 2012;7, e35511.

8. Sindhava VJ, Scholz JL, Stohl W, Cancro MP. APRIL mediates peritoneal B-1 cell homeostasis. Immunol Lett. 2014;160:120-7.

9. Mohr E, Serre K, Manz RA, Cunningham AF, Khan M, Hardie DL, Bird R, MacLennan IC. Dendritic cells and monocyte/macrophages that create the IL-6/APRIL-rich lymph node microenvironments where plasmablasts mature. J Immunol. 2009;182:2113-23.

10. Stein JV, Lopez-Fraga M, Elustondo FA, Carvalho-Pinto CE, Rodriguez D, Gomez-Caro R, De Jong J, Martinez AC, Medema JP, Hahne M. APRIL modulates B and T cell immunity. J Clin Invest. 2002;109:1587-98.

11. Rennert P, Schneider P, Cachero TG, Thompson J, Trabach L, Hertig S, Holler N, Qian F, Mullen C, Strauch K, et al. A soluble form of B cell maturation antigen, a receptor for the tumor necrosis factor family member APRIL, inhibits tumor cell growth. J Exp Med. 2000;192:1677-84.

12. Marsters SA, Yan M, Pitti RM, Haas PE, Dixit VM, Ashkenazi A. Interaction of the TNF homologues BLyS and APRIL with the TNF receptor homologues BCMA and TACl. Curr Biol. 2000;10:785-8.

13. Wu Y, Bressette D, Carrell JA, Kaufman T, Feng P, Taylor K, Gan Y, Cho YH, Garcia AD, Gollatz E, et al. Tumor necrosis factor (TNF) receptor superfamily member TACl is a high affinity receptor for TNF family members APRIL and BLyS. J Biol Chem. 2000;275:35478-85.

14. Kimberley FC, van Bostelen L, Cameron K, Hardenberg G, Marquart JA, Hahne M, Medema JP. The proteoglycan (heparan sulfate proteoglycan) binding domain of APRIL serves as a platform for ligand multimerization and cross-linking. Faseb j. 2009;23:1584-95.

15. Lee SM, Kim EJ, Suk K, Lee WH. BAFF and APRIL induce inflammatory activation of THP-1 cells through interaction with their conventional receptors and activation of MAPK and NF-kappaB. Inflamm Res. 2011; 60:807-15.

16. Xu J, Ding WF, Shao KK, Wang XD, Wang GH, Li HQ, Wang HM. Transcription of promoter from the human APRIL gene regulated by Sp1 and NF-kB. Neoplasma. 2012;59:341-7.

17. Osorio C, Chacon PJ, White M, Kisiswa L, Wyatt S, Rodriguez-Tebar A, Davies AM. Selective regulation of axonal growth from developing hippocampal neurons by tumor necrosis factor superfamily member APRIL. Mol Cell Neurosci. 2014;59:24-36.

18. Deshayes F, Lapree G, Portier A, Richard Y, Pencalet P, Mahieu-Caputo D, Horellou P, Tsapis A. Abnormal production of the TNF-homologue APRIL increases the proliferation of human malignant glioblastoma cell lines via a specific receptor. Oncogene. 2004;23:3005-12.

19. Kim KS, Park JY, Jou I, Park SM. Functional implication of BAFF synthesis and release in gangliosides-stimulated microglia. J Leukoc Biol. 2009;86:349-59.

20. Soderblom C, Luo X, Blumenthal E, Bray E, Lyapichev K, Ramos J, Krishnan V, Lai-Hsu C, Park KK, Tsoulfas P, Lee JK. Perivascular fibroblasts form the fibrotic scar after contusive spinal cord injury. J Neurosci. 2013;33:13882-7.

21. Basso DM, Fisher LC, Anderson AJ, Jakeman LB, McTique DM, Popovich PG. Basso Mouse Scale for locomotion detects differences in recovery after spinal cord injury in five common mouse strains. J Neurotrauma. 2006;23:635-59.

22. Lytle JM, Wrathall JR. Glial cell loss, proliferation and replacement in the contused murine spinal cord. Eur J Neurosci. 2007;25:1711-24.

23. Donnelly DJ, Popovich PG. Inflammation and its role in neuroprotection, axonal regeneration and functional recovery after spinal cord injury. Exp Neurol. 2008;209:378-88.

24. Bartholdi D, Schwab ME. Expression of pro-inflammatory cytokine and chemokine mRNA upon experimental spinal cord injury in mouse: an in situ hybridization study. Eur J Neurosci. 1997:9:1422-38.

25. Chang $Y$, Jia $X$, Sun $X, X u S$, Wu Y, Zhang L, Wei W. APRIL promotes proliferation, secretion and invasion of fibroblast-like synoviocyte from rats with adjuvant induced arthritis. Mol Immunol. 2015;64:90-8.

\section{Submit your next manuscript to BioMed Central and we will help you at every step:}

- We accept pre-submission inquiries

- Our selector tool helps you to find the most relevant journal

- We provide round the clock customer support

- Convenient online submission

- Thorough peer review

- Inclusion in PubMed and all major indexing services

- Maximum visibility for your research

Submit your manuscript at www.biomedcentral.com/submit
Biomed Central 